This is a preprint or reprint of a paper intended for presentation at a conference. Because changes may be made before formal publication, this is made available with the understanding that it will not be cited or reproduced without the permission of the author.

\title{
A UNIQUE POWER SYSTEM FOR THE ISS FLUIDS AND COMBUSTION FACILITY
}

\author{
David A. Fox*, Hamilton Sundstrand, Rockford, IL \\ Mark D. Poljak ${ }^{\dagger}$, NASA Glenn Research Center, Cleveland, OH
}

\begin{abstract}
Unique power control technology has been incorporated into an electrical power control unit (EPCU) for the Fluids and Combustion Facility (FCF). The objective is to maximize science throughput by providing a flexible power system that is easily reconfigured by the science payload.
\end{abstract}

Electrical power is at a premium on the International Space Station (ISS). The EPCU utilizes advanced power management techniques to maximize the power available to the FCF experiments. The EPCU architecture enables dynamic allocation of power from two ISS power channels for experiments. Because of the unique flexible remote power controller (FRPC) design, power channels can be paralleled while maintaining balanced load sharing between the channels. With an integrated and redundant architecture, the EPCU can tolerate multiple faults and still maintain FCF operation.

It is important to take full advantage of the EPCU functionality. The EPCU acts as a buffer between the experimenter and the ISS power system with all its complex requirements. However, FCF science payload developers will still need to follow guidelines when designing the FCF payload power system. This is necessary to ensure power system stability, fault coordination, electromagnetic compatibility, and maximum use of available power for gathering scientific data.

\section{INTRODUCTION}

Designing an experiment for the ISS is a complex job. The last thing the experimenter wants to worry about is the electrical power. If the experiment interfaces directly with ISS power, there are a myriad of specifications that must be read and understood. (See Figure 1) The equipment must be designed, tested, and qualified to meet requirements for conducted electrical magnetic interference (EMI), input impedance, power quality, electrical transients, inrush current, and reverse energy. In addition, long duration experiments must be compatible with constantly changing power availability and allocation issues.

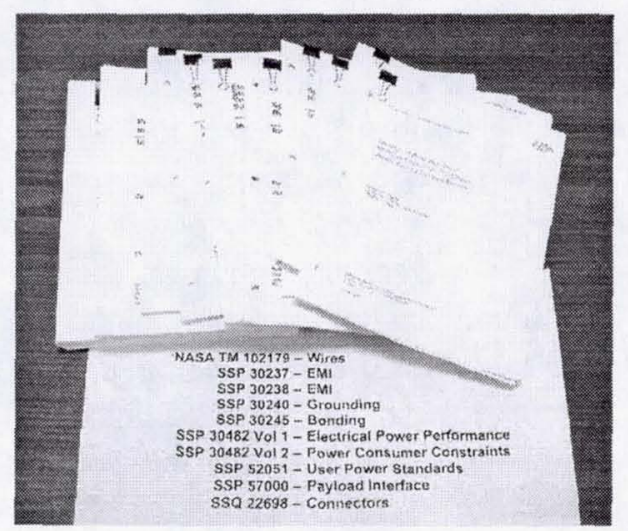

Figure 1

ISS Electrical Power Specifications

For the experimenter using the FCF, all these requirements are taken care of by the EPCU. The EPCU provides the interface between ISS power

\footnotetext{
*Advisory Engineer (David.Fox@hs.utc.com)

† Aerospace Engineer (Mark.D.Poljak@grc.nasa.gov)

Copyright (C) 2001 by the American Institute of Aeronautics and Astronautics Inc. All rights reserved.
} 
and the user while providing protection for both. In addition, advanced power management controls in the EPCU provide increased levels of reliable power for experimental payloads. The EPCU also limits effects of experiment loads on the ISS power system. Fault currents are limited and not passed back to the power source. The EPCU also provides load sharing and priority load shedding internally, reducing the power management load burden on the ISS system.

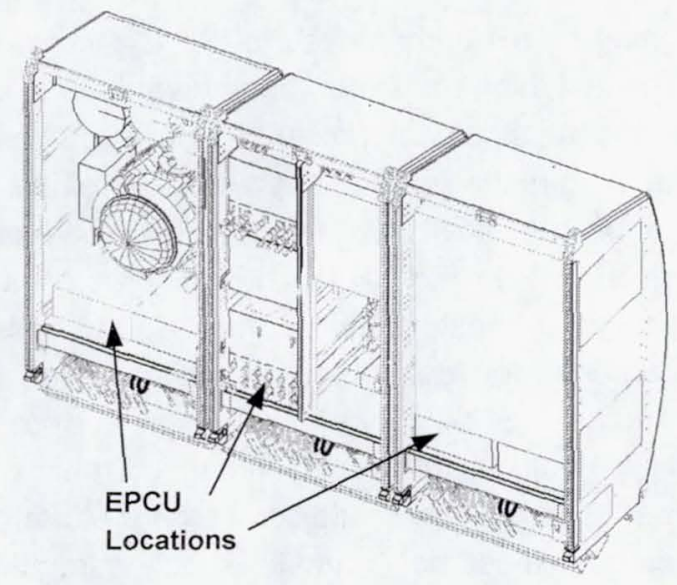

Figure 2

Fluids and Combustion Facility Racks

Figure 2 shows the location of EPCUs in the FCF rack assembly on the ISS. Each EPCU provides power for its rack. Experiments connect to the front of the EPCUs for $28 \mathrm{Vdc}$ power. The front connections allow reconfiguration as required for each unique combination of experiments. $120 \mathrm{Vdc}$ power for fixed rack facilities is provided at the back of the EPCU for safety considerations. Communication to the EPCUs for control and monitoring is accomplished by means of a Mil-Std 1553 Data Bus.

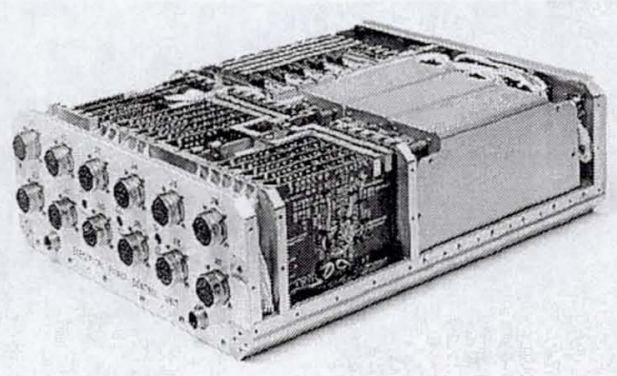

Figure 3 Electrical Power Control Unit

\section{EPCU DESCRIPTION}

A picture of the EPCU is shown in Figure 3. The unit consists of 25 major printed wiring assemblies and three DC/DC converters, all mounted on a water cooled baseplate. The EPCU converts the $120 \mathrm{Vdc}$ secondary power to the $28 \mathrm{Vdc}$ required by most experiments.

The Mil-Std-1553 commands to the EPCU are protected by a Load-Execute protocol which allows a command to be verified before it is executed. This protocol, plus the robust error detection scheme built into the data bus itself, reduces data transmission errors to an extremely low level.



Figure 4

EPCU Block Diagram

As shown in the block diagram of Figure 4, there are (48) $28 \mathrm{Vdc}$ outputs rated at $4 \mathrm{~A}$ each. In addition, there are (6) $120 \mathrm{Vdc}$ outputs rated at $4 \mathrm{~A}$ each for higher power loads. These outputs are controlled and protected by means of FRPCs. In accordance with the ISS guidelines, the $120 \mathrm{Vdc}$ inputs of the EPCU are isolated from chassis and each other. The EPCU provides the single point ground for the $28 \mathrm{Vdc}$ output circuits, which should be isolated at the loads.

Figure 5 shows the location of the major power components of the EPCU as well as the power ratings of the inputs and outputs. The EPCU contains several advanced power management features as described below. 


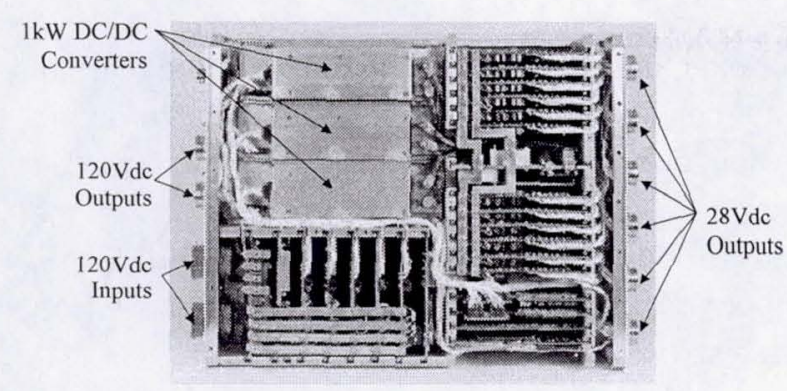

(2) Inputs: $120 \mathrm{Vdc}-50 \mathrm{~A}$

(6) Outputs: $120 \mathrm{Vdc}-4 \mathrm{~A}$

(48) Outputs: $28 \mathrm{Vdc}-4 \mathrm{~A}$

Figure 5

EPCU Power Components

\section{High Efficiency}

There are three $120 \mathrm{Vdc}$ to $28 \mathrm{Vdc}$ converters in the EPCU, each rated at $1000 \mathrm{~W}$. Each of these converters operates at a peak efficiency of over $94 \%$ and over $93 \%$ from 10 to $100 \%$ load as shown in Figure 6. This high efficiency level is approximately $10 \%$ better than most small power supplies available at the power levels needed for FCF experiments. The result is more power for the users. Another important benefit of higher efficiency operation is the reduced thermal load in the FCF rack. The EPCU requires less than half the cooling needed for typical equivalent power supplies

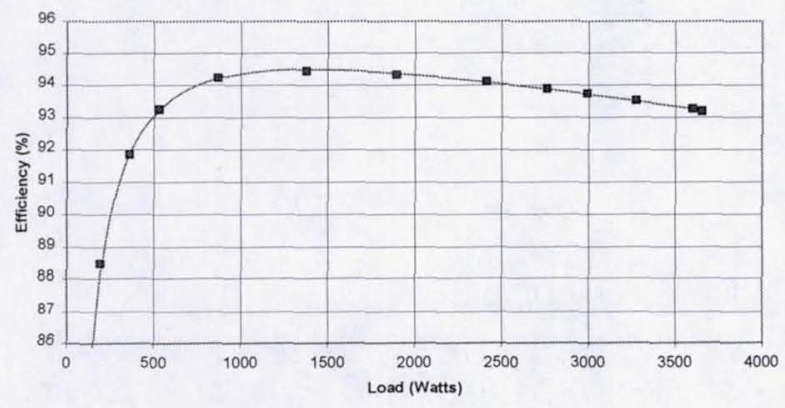

Figure 6 EPCU Converter Efficiency

Load Sharing

The three converters and the (6) $120 \mathrm{~V}$ outputs of the EPCU may be powered from either of two ISS power channels available to the FCF. Power input allocations are downloaded by means of the MilStd 1553 interface. When the power allocation limit for an input is approached, the converters, which are paralleled, adjust their power sharing ratio automatically to reduce the input power on the loaded channel, maintaining the input power within the allocated limit. This type of event may occur many times during a long experiment. The EPCU provides the load sharing capability automatically, without intervention from the ISS power system, and without disturbing the connected experiments. The net result is greater increased power reliability for the experiments and easier load management.

\section{Load Shedding}

If the load power demand increases beyond the allocation, or the input allocation is reduced, the EPCU provides for automatic load shedding of the $120 \mathrm{Vdc}$ and $28 \mathrm{Vdc}$ outputs per downloaded priorities. Load shedding continues until the input power is reduced below the allocation limit. The load sharing and load shedding features allow the EPCU to provide power right up to the allocation limit at all times, without requiring excessive headroom on the loading to prevent exceeding the power allocations. Thus, more power is available for the experiments for a given allocation. Prority trip levels from 0 to 15 can be downloaded for every FRPC output. Level 15 is the first to trip off when the allocation is exceeded. Level 0 is the "no trip" level that disables the priority trip function for the channel.

An example of load shedding is shown in Figure 7. The multiple traces track the events that occur when one channel of input power for the EPCU is disabled. The lower trace (lout21) shows the input current from the failed source dropping from 17.0A to 0 . The next trace (DDCU7out) is the input voltage from the remaining power source of the EPCU provided from a ISS power system DDCU (DC to DC Converter Unit). The increased load on the remaining input causes the input voltage to decay from $122.24 \mathrm{~V}$ to a reduced level over a period of $200 \mathrm{msec}$. The steady trace through the middle of the picture (Vbus) shows the converter output voltage which is unchanged at about $28 \mathrm{~V}$ 


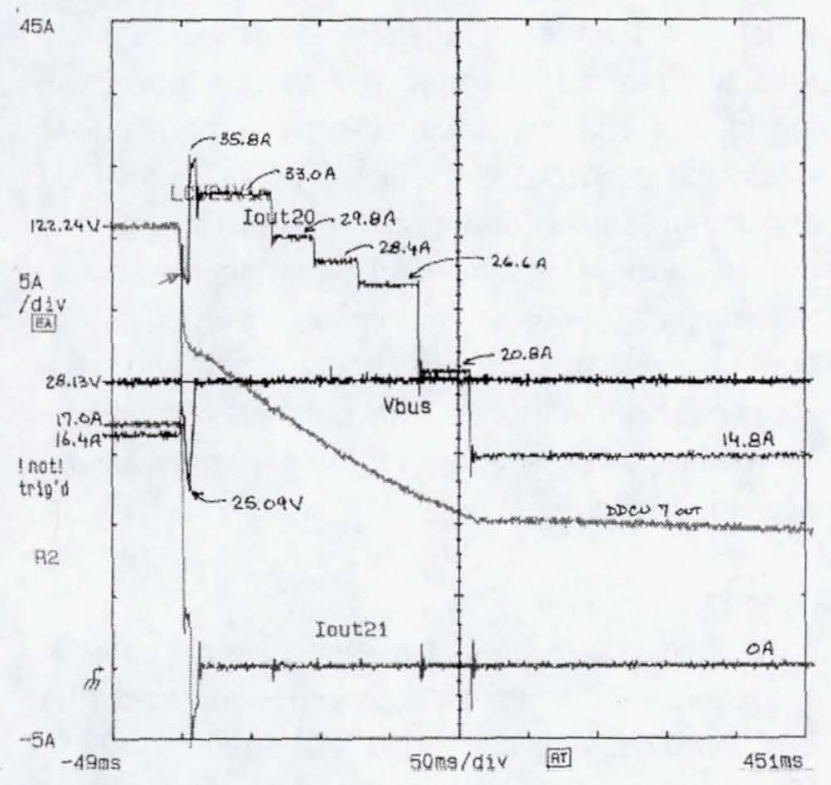

Figure 7

Load Shed Example

throughout the event except for a short, three volt transient caused by the loss of one channel of input power.

The top trace (lout20) shows the increase in input current for the remaining power source, which picks up the power dropped by the failed input, increasing from $16.4 \mathrm{~A}$ to a peak of $35.8 \mathrm{~A}$. For this experiment, the input allocation was set to $18 \mathrm{~A}$, so the priority trip circuits begin to remove loads, according to the preset load priorities. The result is several stairstep reductions of input current, until the last step reduces the current below the $18 \mathrm{~A}$ allocation to $14.8 \mathrm{~A}$, when the priority tripping stops and the remaining loads are maintained. When properly set up, the power demand on the overloaded channel will be reduced within the overload timing capability of the DDCU power source for the EPCU.

It must be explained that the load shedding feature is not only designed to compensate for the loss of an input power channel. It will also operate if the EPCU power allocations are reduced during operation. The load shedding rates can be adjusted by proper selection of the priority levels.
For example, large heating loads with long thermal time constants may be occasionally interrupted without disturbing experiments. Such loads can be assigned priority levels such as 15 which is the first to be tripped. Other, more critical loads, should be assigned lower number priorities which are less likely to be interrupted.

The timing between trip levels may be adjusted by skipping priority levels. The longer time between trips will give the system more time to stabilize after each event.

\section{Flexible Remote Power Controllers}

The FRPCs control the $28 \mathrm{Vdc}$ and $120 \mathrm{Vdc}$ outputs of the EPCU. Each output is current limited at 4.2 to $4.6 \mathrm{~A}$ by active circuits in the FRPC. An inverse protective trip is provided as a function of the voltage across the switch. The combination of current limiting and voltage trip functions provides advantages that earn the name Flexible RPC.

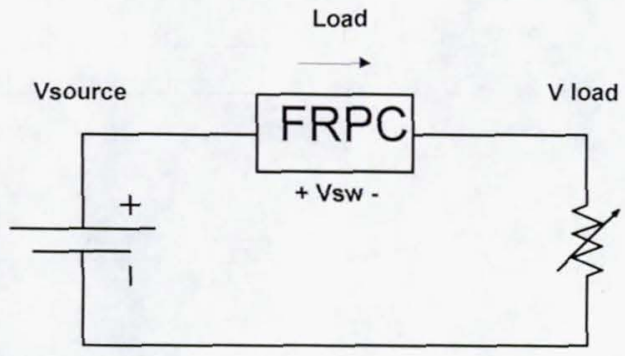

Figure 8

FRPC Operation

\section{Switch Voltage Drop}

The switch voltage drop is the voltage across the switch element of the FRPC. It is the difference between the line voltage and the voltage across the load, as shown in Figure 8. The load voltage is determined by the load impedance and the current limit value of the FRPC. The power dissipation of a current limited FRPC is proportional to the voltage across the switch, because the current is constant during an overload. To protect the switching device, the trip time must be shorter for short circuits or heavy overload conditions where the switch voltage drop is large. This suggests an overload trip function 
with time inversely proportional to the switch voltage drop. Such a trip curve is shown in Figure 9. The shape of the curve is tailored to match the transient thermal impedance of the solid state switch element. This allows maximum use of the switch thermal capability. The curve is designed for a $120 \mathrm{Vdc}$ system.

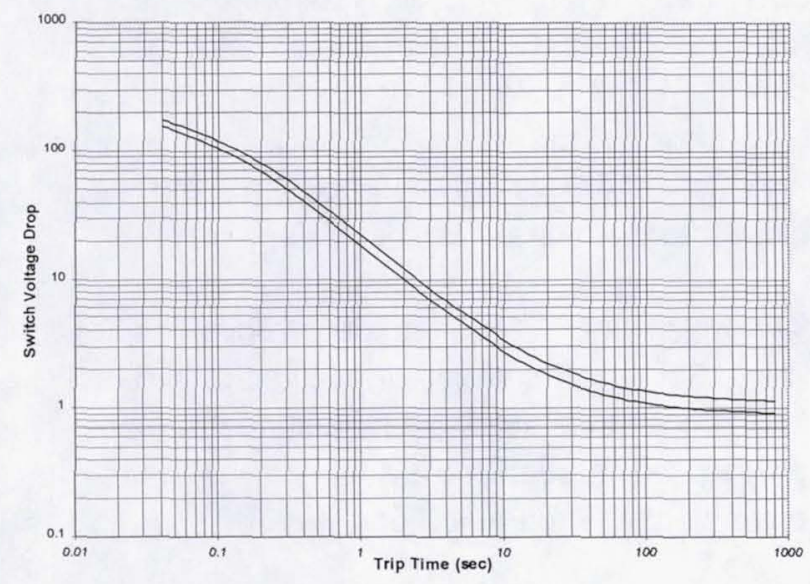

Figure 9

\section{Switch Voltage Drop Trip Characteristic}

With a short circuit across the FRPC, the switch voltage drop will be $120 \mathrm{~V}$ and the trip time as read from the trip curve will be a little less than 100 milliseconds. At $28 \mathrm{~V}$, the trip time would be about 700 milliseconds. This points out an important feature of the the switch voltage drop protection scheme - it allows an FRPC to operate at any line voltage within its rating.

The inverse characteristic of the trip curve also allows the FRPC to charge a much larger capacitive load than the equivalent fixed trip time current limiting RPC, such as used on the Space Shuttle. For the curve of Figure 9, up to four times the value of capacitance can be charged. At $120 \mathrm{Vdc}$, each FRPC channel will charge 10,000 microfarads with no other load, or 5,000 microfarads in parallel with a $4 \mathrm{~A}$ resistive load. At $28 \mathrm{Vdc}$, each FRPC channel will charge 200,000 microfarads with no other load, or 100,000 microfarads in parallel with a $4 \mathrm{~A}$ resistive load.

\section{FRPC Reverse Current}

If the input power source for an FRPC is faulted, reverse current will flow back through the FRPCs from energy stored in the load capacitance. This current will not damage the FRPC FET switch elements, but could blow the failsafe fuses incorporated into the designs. For this reason, $120 \mathrm{Vdc}$ loads with more than 3000 microfarads or $28 \mathrm{Vdc}$ loads with more than 4000 microfarads should add a series diode to their input circuits to block reverse current flow.

\section{FRPC Parallel Operation}

The FRPC design allows the outputs to be paralleled to provide for higher power loads. Any number of FRPC outputs may be paralleled. The flexibility of the design is a perfect match for the FCF operation which requires re-configuration for every one of the scores of experiments to be supported over the lifetime of the facility. The flexibility of the FRPCs allows control of single 4A channels or parallel operation of over half the output channels together with a current capability of over 100A (limited by the converter ratings.) The parallel operation of power channels is controlled by jumpers installed in the loads or wire harnesses for each specific load configuration.

Figure 10 shows the power and programming connections for each group of $28 \mathrm{Vdc}$ outputs within one of the 12 front panel connectors. There are four power output connections (the return connections are not shown). There is a matching set of four jumper connections as well as an Initial Position programming pin.

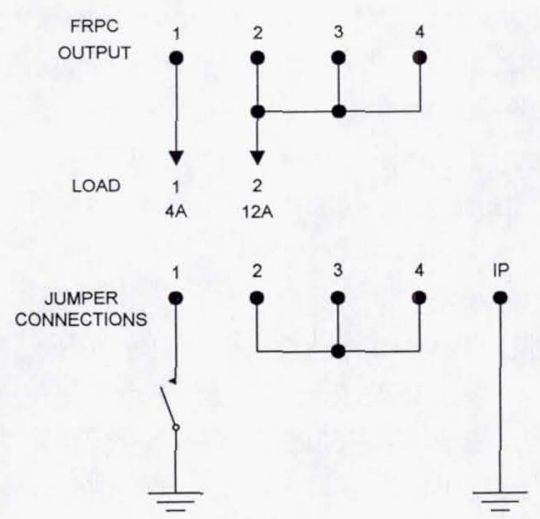

Figure 10

FRPC Power and Programming Connections 
For the example shown in Figure 10, channel 1 is connected by itself to provide a single $4 \mathrm{~A}$ output. Channels 2 through 4 are paralleled to provide a $12 \mathrm{~A}$ output. The jumper connections for channels 2 through 4 are interconnected to match the power outputs. These connections cause the three channels to operate at the same time, in response to a control signal on channel 2. The three channels will also trip together in case of an overload.

It is possible to utilize the parallel jumpers in another fashion. The FRPC power outputs may not be paralleled, but connected to the same experiment, such as various power supplies or heaters. In this case the programming jumpers may be connected to force the outputs to switch at the same time or trip off together if there is a fault.

The IP jumper pin is shown connected to ground. This pin programs the state of the channel 1 of the group when power is applied to the EPCU. With the pin grounded, channel 1 will automatically be turned on at power up. This operation is critical for the EPCU, where output power of the EPCU is used to power the computer that controls the EPCU. Without this feature, the system could not be started.

The jumper on channel 1 shows a switch connected to ground. Each of the four jumper pins responds to a ground connection by tripping off the associated FRPC. This allows a load to activate protection for itself without the necessity of providing an additional input power switch or circuit breaker. This type of protection could be provided by a simple thermostat switch to short the jumper to ground.

\section{Trip Coordination}

In the EPCU, only one level of fault protection is provided for the $28 \mathrm{Vdc}$ outputs. Each output is provided by one FRPC connected to the $28 \mathrm{Vdc}$ converter output. The $120 \mathrm{Vdc}$ outputs are also connected to individual FRPC outputs, but the $120 \mathrm{Vdc}$ inputs are provided by the ISS RPCMs. The following trip coordination discussion is included primarily to demonstrate the advantages that FRPCs can provide in large complex systems where there may be several levels of protection and series connection of protective devices.

Fuses and circuit breakers are difficult to coordinate in this type of system. A fault at the load end of the distribution system may generate currents in all protective devices in series all the way back to the source. Coordination requires that, for any level of fault current, the device closest to the fault will trip before any other.

Current limiting FRPCs aid trip coordination in two ways. First, fault currents are limited by the FRPC to a known, safe value. High fault currents are not propagated through the system to the source. In other words, "The Fault Stops Here."

Second, the switch voltage drop trip characteristic simplifies trip coordination as shown in the following example.

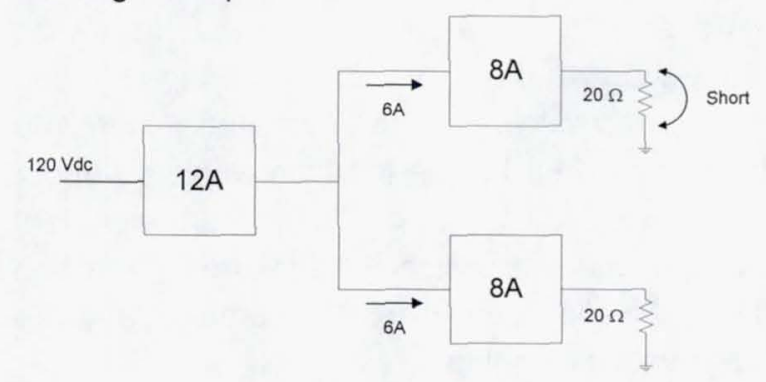

Figure 11

Trip Coordination Example

The example shown in Figure 11 is a $120 \mathrm{Vdc}$ branch with three current limited FRPCs protecting two loads. The two $20 \mathrm{ohm}$ loads carry six amps each and are protected by $8 \mathrm{~A}$ FRPCs. The two FRPCs are powered by one 12A FRPC which is $100 \%$ loaded. The $12 \mathrm{~A}$ FRPC is close to tripping and should be replaced with a higher rated unit. This example will show how, even with this system design, where the 12A FRPC is fully loaded, the proper FRPC will trip.

If a short occurs, as indicated in the diagram, two of the FRPCs go into current limiting, with the results shown in Figure 12. 


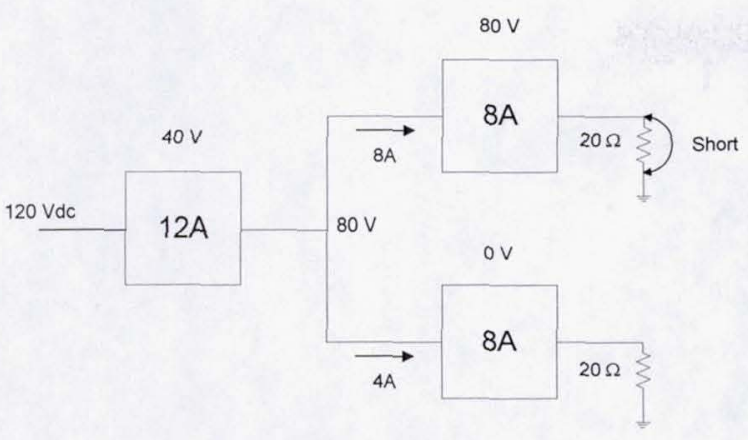

Figure 12

Trip Coordination Example Results

The faulted FRPC limits the current to $8 \mathrm{~A}$. It is fed by the 12A FRPC, which is also current limiting, leaving only $4 \mathrm{~A}$ for the lower load. A current of $4 \mathrm{~A}$ into the $20 \mathrm{ohm}$ load produces $80 \mathrm{~V}$ as shown on the diagram.

Now, which of the two FRPCs will trip first? The 12A FRPC has a switch voltage of $120-80=$ $40 \mathrm{~V}$. The trip curve of Figure 9 shows that it will trip in about 450 milliseconds. The upper $8 \mathrm{~A}$ FRPC has a switch voltage of $80 \mathrm{~V}$ which will cause it to trip in 200 milliseconds. Therefore the faulted $8 \mathrm{~A}$ FRPC will trip first. Because the 12A FRPC was fully loaded before the fault, the only system effect of the fault will be the loss of load when the top FRPC trips. The lower load will see a brown-out to $80 \mathrm{~V}$ for 200 milliseconds.

\section{Output Voltage Abnormal Transients}

When things go wrong, the $28 \mathrm{Vdc}$ outputs of the EPCU can experience values outside the steady state limits ( 27 to $29 \mathrm{Vdc}$ ) for some period of time. Output voltage of the converters is continuously monitored. If excess voltage is generated by one of the converters, that converter is tripped off.

If the output of a $28 \mathrm{Vdc}$ FRPC is faulted, the voltage will be zero while the fault is maintained. If the fault clears before the FRPC trips, the voltage will recover. If not, the output will trip off.

Figure 13 shows the envelope of possible voltage outputs from the EPCU developed from the converter overvoltage trip curve and the FRPC voltage trip characteristic.

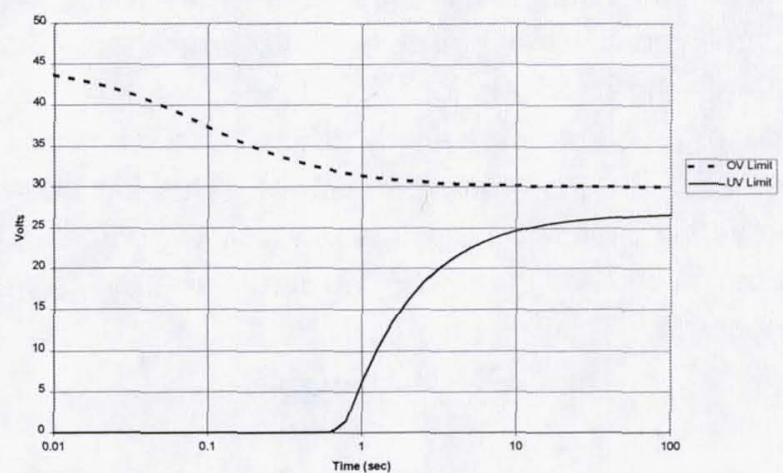

Figure 13 28V Abnormal Limits

Fuses, Circuit Breakers, and SSPCs

Now that operation of the FRPCs has been explained, let's go back and compare with other protective devices, such as fuses and circuit breakers. Most other devices operate with an inverse trip characteristic similar to Figure 8 , but based on current instead of voltage. Right away this means that large overload currents must flow to cause the protective device to open. The large currents cause voltage transients on the power system that affect every load on the system. The magnitude of fault currents that can flow is limited only by the impedance of the source, connections, and wiring

Turning on a large capacitive load will cause the same currents as application of a fault. It is difficult to prevent a trip of the protective device in this situation. The large currents can cause upstream devices to trip first, unless the characteristics are carefully matched and coordinated. In general, this means that the trip curve for each upstream device must always lie above (higher current) and to the right (longer time) of the downstream device, for every current and time combination. The hierarchy of protection may include combinations of fuses, circuit breakers, and SSPCs in series. The trip coordination example of Figures 11 and 12 would almost certainly trip the wrong device, in spite of careful matching of characteristics. 
In general, conventional protective devices, such as fuses and circuit breakers, may only be paralleled if they are carefully matched. So paralleling channels for larger loads may prove to be a problem, particularly if the fuse or circuit breaker is temperature sensitive. This limitation severely limits the applicability for experimental use such as the FCF, where flexibility is a necessity.

\section{CONCLUSIONS}

The power management capabilities of the EPCU and the protection capability of the FRPCs clearly provide many advantages to the experimenter using the FCF. The EPCU also alleviates the workload of the ISS power management system, automatically handling allocation problems and stopping fault propagation at the source.

More power is available to the users, with increased reliability. This increases the probability of successful completion of long duration experiments with critical power demands.

The benefits of the FRPC concept can be implemented in other power systems at any level at the source, loads, or anywhere in the distribution system - to limit fault propagation and improve fault coordination.

\section{ACKNOWLEDGEMENT}

The development of the EPCU was supported by the NASA Glenn Research Center. Information about the NASA Fluids and Combustion Facility program can be obtained from:

Robert Zurawski

ISS FCF Project Manager

NASA GRC

21000 Brookpark Road

Cleveland, $\mathrm{OH} 44135$

(216) 433-3932 